\title{
Setting a foundation: underlying values and structures of health promotion in primary health care settings
}

C James Frankish Institute of Health Promotion Research, Faculty of Graduate Studies, Department of Health Care and Epidemiology, Faculty of Medicine, University of British Columbia, Vancouver, BC, Canada, Glen Moulton Institute of Health Promotion Research, University of British Columbia, Vancouver, BC, Canada, Irving Rootman Faculty of Human and Social Development, University of Victoria, Victoria, BC, Canada, Carol Cole and Diane Gray Institute of Health Promotion Research, University of British Columbia, Vancouver, BC, Canada

\begin{abstract}
Jurisdictions around the world have articulated a need for development of an integrated health care system with increased emphasis on primary health care and incorporation of the principles and practices of health promotion. To date, the medical model has been the default model of care. Yet, treatment alone is unlikely to have marked effects on health inequities or health status. This article presents and discusses two foundational dimensions (values, structures) of a health promotion philosophy and approach in primary health care settings. We propose a strategy and framework to support practical and attainable action. Our article (the first of a series of two) is based on a literature review, validation by key experts and a national survey of Canadian primary health care settings. We argue that fundamental philosophical values provide a foundation for health promotion in primary health care. These values should be reflected in the structures that create a supportive environment for health promotion. Our remaining three domains (strategies, processes, outcomes) are presented in a second following article. Based on values and structures, we conclude that subsequent strategies (interventions), processes (client- and community-centred care), and desired health promotion outcomes (intended or unintended) may be achieved.
\end{abstract}

Key words: health care reform; health inequities; health promotion; population health; primary health care

Received: May 2004; accepted: December 2005

\section{Introduction}

Jurisdictions around the world (McElmurry et al., 2002), such as Australia (Victorian Department of Human Services, 2000), Britain (Adams et al., 2001; Gillam and Schamroth, 2002), Canada (Marriott and Mable, 2000), and New Zealand (King, 2001; Ministry of Health, New Zealand, 2003), have articulated the need for development of an integrated health care system with increased emphasis on

Address for correspondence: Glen Moulton, Institute of Health Promotion Research, University of British Columbia, Room 411, LPC Building, 2206 East Mall, Vancouver, BC, Canada V6T $1 Z 3$. Email: moulton@interchange.ubc.ca primary health care, and incorporation of principles and practices of health promotion. Our article elucidates the requisite dimensions of a health promotion philosophy and approach in primary health care settings. We provide a strategy and framework to support practical and attainable action. Our aim is to provide clarity and an operational definition to health promotion so that it can be practically addressed.

\section{Context and rationale}

In recent decades, health promotion has developed in isolation and functioned separately from primary 
care. More recently, the philosophy underpinning health promotion has become accepted more widely. Despite this apparent increase in awareness of and predisposition toward a health promotion philosophy, health promotion remains largely on the fringes of primary health care. This is due to the preventive and perceived, 'non-urgent' nature of health promotion when compared with clinical/medical care (Ziglio et al.,2000). The medical model has been the default model of care (McElmurry and Keeney, 1999). Treatment alone is unlikely to have marked effects on health inequities. It is only recently that many jurisdictions and health professionals have recognized that a reduction in inequalities and a closing of the gap in health status requires greater integration of health promotion in primary health care (HP in PHC) (Keleher, 2001; Ministry of Health, New Zealand, 2003). It has been estimated that health services only contribute about a fifth of health improvement (Evans et al., 1994). Today's health problems demand a health promotion approach. Health improvement mainly occurs through changes in the social, economic, and cultural impacts on communities (King, 2001). A narrow technical approach is inadequate for intervening in health problems that have a social, economic, behavioural and/or psychological basis. With increased focus on health promotion, health services may be a more effective determinant of health.

While HP in PHC is recognized as reasonable/ beneficial, it is not easy to achieve. There remains a disparate understanding of health promotion concepts and the inherent diversity of potential applications (McElmurry and Keeney, 1999). There is a need for a strategic approach to guide programme planning, implementation, evaluation, education and research. Stakeholders need to make their conceptual framework/understandings of health promotion more explicit and tangible.

Health promotion research in primary health care often focuses on only one or two of the many components. This contributes to the maintenance of diverse and fragmented perspectives. It is critical that we identify and define terms and concepts underpinning HP in PHC. We can do so by differentiating it from the more predominant diseasefocused model. By operationalizing the breadth and diversity of HP in PHC, settings may be able to develop and sustain it. This fundamental change requires a philosophical paradigm shift as well as practical implementation.

\section{Definition of primary health care}

We recognize that the terms 'primary care' and 'primary health care' are often used interchangeably, and sometimes have different meanings for different people. We use the definition of primary health care suggested by the National (Canadian) Forum of Health: 'The care provided at the first level of contact with the health care system, the point at which health services are mobilized and coordinated to promote health, prevent illness, care for common illness, and manage health problems' (1998: 22). This definition includes a focus on a primary (medical) care model, usually provided by family physicians, and a broader concept that encompasses a range of health/social services provided through multidisciplinary teams. Primary health care is the natural entry-point to reorient a health system towards health promotion.

Primary health care providers include chiropractors, dentists, dieticians, family physicians, health educators, midwives, nurses, nurse practitioners, optometrists, pharmacists, physiotherapists, psychologists, and social workers. Settings in which a health promotion philosophy/approach could be expanded include community health centres, emergency rooms, outpatient clinics, pharmacies, private solo or group practices, public health units, health vans, outreach centres, store-front settings, and walk-in clinics.

While there has been considerable work done on 'health promoting hospitals,' institutionalized acute-care settings were not the focus of this project. While some settings may be more conducive to adopting a health promotion philosophy/practice than others, we argue strongly that there is a role and responsibility for providers in every setting to implement health promotion.

\section{Definition of health promotion}

Despite some common definitions of health promotion (World Health Organization (WHO), 1986), we found that many people associated with primary health care continue to understand the term 'health promotion' differently. This may contribute to continuing confusion and a lack of consensus. Our article highlights the need for great attention and clarity regarding the meaning of HP in PHC. It can be a complex, sophisticated, vague and variable 
phenomenon within primary health care. More often than not, health promotion is understood too narrowly. Health education is often seen as the sum total of health promotion. This unfortunate perspective is common to health decision-makers looking for simplistic behaviour change or one size fits all interventions.

There are many published definitions of health promotion. The most widely cited and used throughout the world is that health promotion is 'the process of enabling people to increase control over, and to improve, their health' (WHO, 1986). An elaboration of this definition has become popular, namely 'the process of enabling [individuals and communities] to increase control over [the determinants of health] and [thereby] improve their health' (Nutbeam, 1998). These definitions have implications for the way in which health promotion is practiced in primary health care settings. They imply that health promotion involves enabling or 'empowering' people to address factors that affect their health. They imply that it does so by increasing their 'control' over these factors or 'determinants'. This might be accomplished by helping them obtain access to needed resources, or by helping them develop personal and collective capacities. The definition also suggests that the desired outcome of health promotion is 'improvement' of health rather than simply its maintenance. It implies that health is a 'positive' concept that people need to strive toward. The use of the terms 'individuals and communities' as a substitute for 'people' in the second definition suggests that health promotion is an enterprise or set of activities that is focused on individuals and the communities where they live. We define community as any group of citizens that have either a geographic, population-based, or self-defined relationship and whose health may be improved by a health promotion approach. Similarly, we employ the term 'client' rather than patient because it de-medicalizes the issue of care, avoids notions of dependence, and reflects a broader cadre of services or activities with which the individual may be involved beyond the simple receipt of treatment.

\section{Research design}

We consulted broadly, using qualitative and quantitative methods, to construct a conceptual framework for HP in PHC that contains the most salient characteristics of HP in PHC from dozens of possibilities. Our research began with an extensive review of the literature in journals using databases, such as MEDLINE, HaPI (Health \& Psychosocial Instruments), CINAHL (Cumulative Index to Nursing \& Allied Health), PubMed, and Embase. We searched the terms 'health promotion,' 'primary health care,' 'primary care,' 'community health centres/centers,' and 'reorienting health services' separately and combined from 1990 to 2004. An Internet search of 620 sites was done combining search terms 'health promotion' and 'primary care' or 'primary health care.' We retrieved a variety of grey literature, that is policy documents and reports. Our review yielded relevant materials from countries throughout the world.

We then employed a modified Delphi technique in which we convened experts to seek their opinions on the relevant characteristics of a conceptual framework of HP in PHC. Our experts included a team of researchers from the Health Reform Working Group of the Canadian Consortium for Health Promotion Research, as well as a multidisciplinary advisory committee of practitioners/policy makers. It included individuals with internationally recognized expertise in health promotion and/ or primary health care. The experts had diverse educational backgrounds, such as nursing, medicine, social work, psychology, and education. They were employed in academia, health administration and/or health service delivery. Based upon the literature review and feedback from experts, an interim conceptual framework was drafted and re-circulated to experts to start the next cycle of seeking input/clarification. This process continued until experts were satisfied with the conceptual framework.

We then sought feedback on our interim framework from additional experts to enhance its trustworthiness. A focus group was held in four Canadian cities (Toronto, Vancouver, Halifax and Edmonton). Forty-eight people participated. They included health researchers/academics, health service practitioners, and policy makers with a variety of educational training.

The final phase of our research was a national survey of primary health care settings to examine the level of importance and activity that professionals and administrators attributed to the various characteristics of the conceptual framework. In the winter of 2003, we conducted a survey of primary 
health care settings in Canada on the perceived importance and reported level of activity of the values, processes, structures, and outcomes of health promotion within their setting. The questionnaire was available online or in hardcopy. It contained quantitative and qualitative questions. Of the 331 English-speaking settings sampled, we obtained a response rate of $52 \%(N=171)(110 / 184$ community health centres, $27 / 50$ public health units, $15 / 48$ family practice units, $4 / 6$ hospital units, and $15 / 43$ nurse practitioner units). This response rate is similar to surveys in other jurisdictions (Baum et al., 1998). Settings were recruited through an Internet search and by contacting national/ provincial organizations of health care professionals and settings. We included provincial and national associations of community health centres, regional health authorities, provincial/territorial Ministries of Health, and university-based family practice units associated with 16 medical schools. Settings were faxed or emailed one to three reminders.

Our initial framework underwent a process of refinement with each successive research phase. The framework evolved substantially. The characteristics within the five domains (values, structures, strategies, processes, and outcomes) were modified until no other aspects of HP in PHC could be identified that were not already included in the framework. Each of the characteristics underwent a cyclical process: continuous discovery to identify patterns; categorizing/ordering data; qualitatively assessing the trustworthiness of data to refine patterns; and synthesizing the themes/concepts into characteristics of the framework (McMillan and Schumacher, 1997).

The underlying foundation of our conceptual framework is rooted in international documents, such as the Ottawa Charter of Health Promotion (WHO, 1986) and the Declaration of Alma-Ata (WHO, 1978). The use of international literature and internationally renowned experts increases the overall credibility and potential transferability of the conceptual framework to jurisdictions throughout the world. At a policy and practical level, the application of the conceptual framework will vary across developed and developing countries, but will also vary within countries and primary health care settings. The conceptual framework presented recognizes the evolving nature of health promotion and primary health care in all countries, regions and settings due to economic, social, cultural and political conditions.

\section{Key domains and characteristics of HP in PHC}

The following section describes our conceptual framework of HP in PHC. The key characteristics are clustered into five domains (values, structures, strategies, processes, and outcomes). Health promotion comprises multiple, interconnected concepts that need to be incorporated into the daily practice of primary health care. We believe that the only way to truly engender health promotion is to integrate all domains and as many characteristics as applicable. The elements of our descriptive domains are neither mutually exclusive nor independent.

First, the philosophical values that provide the foundation for HP in PHC are described. Next, we discuss how values might manifest in specific structures. Structure describes the characteristics of a primary care setting that create a 'supportive environment' for health promotion. Our purpose is to present and discuss these two dimensions (values, structures) as a foundation for HP in PHC settings. The remaining domains (strategies, processes, outcomes) are presented in Moulton et al., 2006.

We offer an operational definition of each characteristic. HP in PHC is most effective when all domains/characteristics are developed and coordinated. The two domains are presented sequentially, but we recognize that the implied 'linearity' of our model belies its true iterative nature. The framework is neither definitive nor prescriptive, but rather enabling since the priorities for individual regions/ settings will vary and need to be adjusted accordingly. No single detailed step by step guide for use by primary care agencies is suggested, but key components for ensuring quality health promotion are outlined. They are deliberately flexible to allow for creative programming, since a 'one size fits all' approach is counterintuitive to health promotion. None of the dimensions within the two domains are unique to health promotion per se. Health promotion is unique precisely because it is an amalgam of values/practices that enhance health. The information provided exemplifies the breadth of the subject area and is condensed into the shortest format possible, using simple language. (This has 
repeatedly been identified as a priority among stakeholders.) Practitioners and decision-makers may require additional information about particular areas of interest to acquire sufficient depth.

\section{Values}

People working in primary care and/or health promotion share common culturally transmitted values regarding health/health services. These values are fundamental in affecting which health issues are addressed, and the strategies that we employ. Values/norms within an organization are often implicit and may be ignored or overlooked in thinking of health promotion.

\section{Broad view of health and its determinants}

Health is influenced by more than just provision of health care. Health promotion subscribes to a positive, multidimensional view of health that focuses on the whole (ie, physical, mental, social) person or the community. It recognizes the role of broad determinants (eg, income, social support, education, employment or working conditions, housing, food security, social environments, physical environments, health practices, coping skills, child development, gender, culture, biology/genetics, health services) in creating/maintaining health and quality of life. These determinants may each interact with one another. We cannot simply promote that individuals change their attitudes and lifestyles, when the environment in which they live and work provides little or no choice or support. Immediate problems of finances, food, and shelter may be so dominant that long-term considerations of health are irrelevant. To better incorporate health promotion values, the practice of primary care must address causal circumstances, not just treatment. Action is based in the social and cultural context.

\section{Optimal health and quality of life for all}

Health is more than the absence of disease or disability; it includes vulnerability to disease and disability. Health promotion is concerned with health problems before they develop or worsen, not only after they appear. Health promotion aims to reduce differences in health status and vulnerability, and to ensure equal opportunities and resources to enable all people to achieve their fullest health potential and quality of life.

\section{Empowerment}

Health promotion is the process of enabling individuals and communities to increase control over, and to improve their health. It demands the meaningful participation of affected individuals in planning, implementation and evaluation of interventions intended to improve their health and quality of life. People cannot achieve their full potential unless they are able to take control of those things that determine their health. Empowerment has a psychological and a community perspective. The former is concerned with self-esteem/selfefficacy, and the latter is concerned with political action. Health promotion emphasizes 'power-with' rather than 'power-over'.

\section{Social justice and equity}

Health promotion is concerned with removing disparities in health and access to its determinants for disadvantaged/at-risk populations. It places a premium on social justice, diversity, fairness, and removal of barriers to equitable participation in aspects of society that influence health and quality of life, including access to health services. Access to health and its determinants should be based on need (and not demand), and attention must be given to people's social realities. While there is a need for self-responsibility and changes in knowledge, attitudes and behaviours, people cannot be reliant upon economic, social or cultural resources they do not possess.

\section{Social and environmental sustainability}

Health promotion subscribes to a 'stewardship' ethic that recognizes responsibility for the health of present/future generations and judicious, sustainable use of human and natural resources. The inextricable links between health (or illness) and people's human/natural environments create a basis for an ecological approach to health. Health promotion protects the interface between the human, natural, social, economic, and built environments, through appropriate land use planning, transportation planning, sanitation, safe water, clean air, safe food supply, and proper nutrition.

\section{Social capital and healthy communities}

Social capital is a concept that recognizes the value and need for a civil society, that a range of social/community circumstances can influence health, and that individual/community health and 
well-being can be affected by the way people relate to each other. Indicators of social capital include community/ civic participation, social relationships, social support, reciprocal activities and levels of trust in others.

\section{Integration}

Health promotion is integrative in three ways: it subscribes to a holistic, systems view of health and quality of life; it seeks to bring together individuals and communities in efforts to maximize their health; and it aims to integrate a health promotion perspective into societal institutions. There is a need for horizontal integration (ie, links between primary health care and other sectors of society) and vertical integration (ie, links between clients, front line service providers and decision leaders and communities, within and across nations).

\section{Appropriate models and methods for accountability}

Interventions designed to influence health/quality of life must be accountable (appropriate, relevant, useful) not only to clients, but for the health of the community and to society as a whole. HP in PHC is a shared responsibility among individuals, professionals, community groups, health service institutions and all levels of government. To date, many of the models, standards and methods for accountability in primary care settings do not fit within a health promotion, community context. All forms of evidence are a social construction. Qualitative and/or quantitative approaches should be used and valued as the situation demands. Health promotion values indigenous knowledge and practices, and seeks to achieve positive societal change while respecting existing cultural groups and communities.

\section{Structures}

The structures domain describes the characteristics of the primary health care setting that create a supportive environment for health promotion. Health promotion will remain marginalized if it is relegated to one person in an organization or only developed as an ad hoc project outside of core structures of primary health care.

Values and intention alone are not sufficient to establish a health promotion approach. Creating organizational structures (supports) builds optimal conditions for success/sustainability of health promotion by ensuring that the programmes, policies, and practices of an organization reflect its purpose, values and objectives. The process of change toward a health promotion approach requires concerted/sustained action made incrementally to allow providers to continue to provide care while changing how they deliver that care (Nova Scotia Department of Health, 2001). By building on successful initiatives in a stepwise, evolutionary manner, health promotion can be brought to the forefront of primary health care. Integrating HP in PHC involves delivery of strategies aimed at specific target populations and health issues (see Moulton et al., 2006), within broader efforts to build the capacity of organizations and communities to promote health.

\section{Resource allocation}

The resource allocation for health promotion has typically been too little for sustainable action (Ziglio et al., 2000). Primary health care organizations need funding that reflects the relative health promotion needs of their populations, taking into account factors such as age, sex, deprivation level, ethnicity, and determinants of health. For example, some jurisdictions allocate a minimum of $20 \%$ of their budgets for health promotion (Victorian Department of Human Services, 2000).

\section{Committed personnel}

Management's commitment to, and understanding of, health promotion is often a first vital step in developing an organization wide approach. The most effective champions are often those in midupper management positions with a sense of leadership, motivation, compromise and appropriate negotiation skills (Marshall and Craft, 2000). A health promotion coordinator can play a vital role in educating/supporting staff, driving organizational initiatives and liaising with community agencies. A case manager can ensure that services received by clients are integrated and appropriate so clients are not required to navigate through a maze of agencies. They may be responsible for team leadership and case coordination. Some jurisdictions require each provider to spend a set portion of their time (eg, 20\%) on health promotion (Swaby and Biesot, 2001).

\section{Human resources development (capacity building)}

Training for health professionals prioritizes acquisition of technical and practical skills. Health 
professionals are being encouraged to practice health promotion as if it were a straightforward and innate phenomenon (Keleher, 2001). Health promotion demands a skill set that is distinct from clinical practice but one that is just as complex and worthy of study and practice. Health professionals, especially physicians, often endorse the principle of prevention, yet nonetheless view it as a low status activity, and may seek to limit their own involvement by delegation to others (Adams et al., 2001). When a non-medical (but health-related) issue is identified, the tendency among health care professionals is to acknowledge it and move on to more clinically related topics where they feel they have specific expertise (Cashman et al., 2001). Human resources development in health promotion includes training and education to enhance knowledge and skills of individuals to enable them to perform effectively while accommodating diversity in differences in backgrounds, clinical practice, educational needs, and learning styles. This may consist of in service training, on the job training/ mentoring, and continuing education.

\section{Intersectoral collaboration}

Cross-cutting health issues cannot be tackled by agencies operating in isolation. Partnerships and coalitions are essential to address social and economic factors that mediate health status differences, and to generate community-based solutions to health problems. Partnerships must be actively sought with organizations that may not have an explicit health focus, and requires co-operation among many levels of stakeholders - national governments, regional governments, local governments, local organizations, private sector, and (potential) clients. Genuine partnerships require moving outside 'zones of comfort' and a substantial degree of commitment and trust among partners.

\section{Accessibility}

Health promotion recognizes a fundamental distinction between availability and accessibility. A core problem in primary care is that many services continue to be inaccessible for marginalized populations. Health/social services should be provided as close as possible to where people live and/or work without socioeconomic, geographic, cultural and other barriers, ensuring that health and social services are available, affordable, appropriate, and acceptable for those whose health needs are greatest. Examples to enhance accessibility include mobile/outreach services, extended office hours, drop-in appointments, translators and appropriate pamphlets.

\section{Accountability}

A process for monitoring progress in a health promotion approach ensures accountability and gives attention to how progress is recorded and reported, to whom, how often, and what actions will be taken if the strategy is facing difficulties or is not implemented (eg, Continuous Quality Improvement). This requires a framework that defines specific areas of responsibility for providers, primary health care settings, and regional boards. These accountabilities should be jointly developed and supported by appropriate infrastructure. They require formal service agreements, periodic reviews, adjustments of expectations, and (re)commitment to stated goals. Incentives, rewards and standards for engaging in quality health promotion practices should be integrated into everyday activities. The accreditation and valuing of health promotion work remains a key issue.

\section{Governance and decision making}

Accountability is achieved by establishing formal responsibility for health promotion within management and a working group or steering committee, and by establishing community (client) advisory committees or a community board of directors. Incorporating health promotion action into performance and accreditation agreements, job descriptions and staff performance appraisals at all levels of the agency also improves responsibility for health promotion.

\section{Communication channels}

While a highly technological health system will not lead to a general improvement in the well-being of communities, information systems can enhance coordination and ensure availability and use of pertinent client information. A common client information system also assists in assessing service impact or programme effectiveness, including health status and population health. A strategy to regularly inform health professionals of the range and location of available/appropriate health promotion services is necessary. Finally, health promotion recognizes that information and technology impact differently on different sectors of society. 


\section{Multidisciplinary teams}

No single practitioner or type of practitioner can meet an individual's health needs completely. Health promotion requires a shift from a provider focus, where responsibilities are distinct and separate, to a client focus, where an integrated, multiservice team of providers and professionals (within and beyond the health sector) work together to address needs. The increasing number of practitioners and their changing roles increases risk of fragmentation of care. Wider expertise is necessary, and a better understanding and flexibility of roles and responsibilities is paramount.

Team building should be a deliberate and ongoing activity to foster collegial interdisciplinary relationships (blurring of professional boundaries) or multidisciplinary teams (involving different professionals who maintain their professional boundaries). Organizational structures should be developed in relation to functional groups or programmes rather than based on disciplines (Pearson and Jones, 1994). Health promotion competencies are more diffuse and eclectic than the more technical/definable competencies used by occupations such as nursing and pharmacy (Keleher, 2001). Developing a common language and sharing of information and expertise requires mentoring, workforce development, dedicated case conference time, and regular meetings.

An effective multi/interdisciplinary team also depends on recruiting individuals with the right mix of skills and knowledge, and the right personalities those that show a willingness to collaborate, a dedication to the service delivery model and to principles of health promotion (Alberta Health and Wellness, 2001). Provider satisfaction centres on role recognition (by themselves and others) and their experiences in the collective (Alberta Health and Wellness, 2001). In smaller teams (of 12 or less), members know each other, are aware of and value each other's skills and interests, and share in setting and achieving goals (Pearson and Jones, 1994).

A minimization of professional dominance ensures that no one group or individual is accorded or takes on a dominant role. At the same time, physicians who are part of a multidisciplinary team that employs a health promotion approach (or are developing new service delivery models) must actively participate in broad planning and development at the community and policy level (Nova Scotia Department of Health, 2001).

\section{Organizational culture}

Although not a tangible structure, an organizational environment conducive to health promotion stresses change, learning and personal growth in a transparent and collaborative manner. Although partnerships and grassroots support are imperative, successful settings focus on internal integration before attempting to encompass partners outside the organization.

\section{Discussion}

To improve the health status in developing and developed countries, a revitalization of the tenets and values of the Ottawa Charter and the Alma-Ata Declaration is needed. Commensurate structures must also be refined and/or developed to provide the 'building blocks' for a health promotion approach. This places renewed emphasis on developing locally defined, primary health care models that are both flexible and adaptable.

There is a clear divide between the concepts of 'health promotion' and 'primary health care' and their implementation since these concepts have not materialized into joint, widespread practical action. This is due in part to a lack of understanding of the intricacies inherent in the definitions. There is also considerable confusion in current health service models about the causation of ill health, the importance of the determinants of health and their requisite translation into intervention strategies. Marshall and Craft (2000) assert, 'much of this confusion arises from the peddling of simple conceptualizations of complex phenomena' (p. 43). Health promotion and primary health care are often conceptualized in a simplistic manner, when in fact they are both complex and sophisticated philosophies which have corresponding practical actions to attain improved health outcomes. Both the terms primary health care and health promotion are understood very differently by many stakeholders which is why a clear elaboration of the terms, and their inherent values and structures, is warranted. These values and structures serve as a catalyst and foundation for HP in PHC.

Both 'primary care' and 'primary health care' refer to the first level of contact people have with the health care system. Conventional primary care (or selective primary health care as it is sometimes referred to as) is individually focused on the person 
seeking care. Such care does not take into consideration the distribution of health problems in the community, or the underlying causes of illness and disease. Most primary care interventions are limited to the most basic provision of curative and preventive services, which are reactive, episodic and brief, and thus health promotion interventions tend to be clinical in nature, such as advice giving. Health promotion in primary (medical) care is reduced to a biomedical and disease focus. As such, the individual is targeted for change rather than the social and environmental conditions that underlie the disease or condition. This conventional or selective approach to primary care is inadequate for intervening in health problems that have socioenvironmental root causes, and reducing health disparities. Results of biomedical, lifestyle health promotion initiatives are often unimpressive and yield only small gains at the individual level.

If primary health care is disguised as primary care, it will loose its significance. Often the term 'primary health care' is used without any reorientation of services as a way of legitimizing service delivery, however, primary health care is more comprehensive than primary care. Primary health care is an approach concerned with health promotion and population health. Primary health care is population focused and understands individuals from the broader context and links interrelated health determinants to health outcomes. Primary health care is proactive, and demonstrates a commitment to causal circumstances of the presenting problems. A health promotion approach in primary health care requires a reorientation of health services that addresses downstream problems and upstream health issues, dealing with health and its maintenance as well as disease and its treatment. A health promotion approach in primary health care pertains to both the individual and the population of individuals. It requires a comprehensive approach employing multiple strategies to have substantial change. Health promotion pays attention to the social, economic and political factors that influence a population's health. A health promotion approach would also address the 'inverse care law' given that needs and demands are clearly divergent with those in greatest need of a given intervention the least likely to avail themselves of it.

Uniting health promotion and primary health care in a common conceptual framework strengthens each paradigm and has the potential to improve the health status of the population and reduce health disparities. Since the Ottawa Charter of Health Promotion (WHO, 1986) and the Declaration of Alma-Ata (WHO, 1978), much of the literature and debate has remained as isolated components of categorical disease prevention and management interventions. To this day, there is a lack of consensus regarding a formal, consistent and integrated approach of health promotion in reorienting health care service. Little progress has been attained in defining an explicit, detailed and commonly agreed upon framework for implementing changes in a systematic fashion (Lopez-Acuña et al., 2000). The current research provides a HP in PHC framework to support practical and attainable action in policy, practice and research.

An obvious starting point is the creation of a vision that makes these values and structures explicit. A broad/inclusive definition of health promotion, and a commensurate vision must be planned and agreed upon by everyone from the board and executive level to the front line practitioners and the community at large. Without a clear mandate for health promotion, it tends to remain at the periphery of service delivery, and programmes tend to be ad hoc, issue based and based on health education, alone. This fragmented approach serves to maintain the marginalization of health promotion.

Through the visioning process, stakeholders may interact with one another to negotiate a new set of shared norms, informal/formal rules of behaviour, develop a sense of ownership and a common bond of the problems to be solved even among the most diverse participants (Alberta Health and Wellness, 2001). The vision becomes a framework for action and describes what health promotion will look like when the vision is implemented. As stakeholders and community needs change, a vision must be refined and regularly communicated. The overall goal is to improve the health of individuals and the community by improving the quality/quantity of health promotion that occurs in primary health care settings. The philosophy and mission of the setting must be compatible with partner agencies to ensure true collaboration over time.

In order to achieve a health promotion approach in primary health care, stakeholders (such as policy makers, practitioners, researchers, citizens) must reflect on the values of health promotion, and develop commensurate structures. The proposed 
framework is comprehensive in nature because it recognizes that multiple stakeholders are responsible for promoting the health, and it should not be simply delegated to physicians or reduced to health education. Efforts at HP in PHC have not been 'wrong' per se, but rather are superficial and fall short, and thus are unlikely to have a substantial effect on population health.

\section{Conclusion}

Increasing the orientation of primary care services towards a health promotion approach will bring conventional primary care closer to primary health care as envisaged by the Alma-Ata Declaration. We have argued that for health promotion to come to fruition in a primary health care setting it must have a solid foundation. This foundation comprises key philosophical values and related structures that create a supportive environment for health promotion initiatives. These values and structures must be implemented on a sustained and comprehensive basis.

This article represents the first of a series of two (see Moulton et al., 2006) articles that present a framework of HP in PHC. The framework has five domains (values, structures, strategies, processes, and outcomes). Moulton et al. (2006) present the corresponding strategies, processes and outcomes that build upon the present values and structures.

\section{Acknowledgements}

The authors wish to thank the Health Reform Working Group of the Canadian Consortium for Health Promotion Research, their coinvestigators, advisory committee, focus group participants, and others who contributed to this work. The work was made possible by a grant from the Canadian Institutes of Health Research.

\section{References}

Adams, C.J., Baeza, J.I. and Calnan, M. 2001: The new health promotion arrangements in general medical practice in England: results from a national evaluation. Health Education Journal 60, 45-58.

Alberta Health and Wellness. 2001: Multi-disciplinary teams: lessons learned. Alberta: Alberta Health and Wellness.
Baum, F., Kalucy, E., Lawless, A., Barton, S. and Steven, I. 1998: Health promotion in different medical settings: women's health, community health and private practice. Australian and New Zealand Journal of Public Health 22, 200-205.

Cashman, S.B., Bushnell, F.K.L. and Fulmer, H.S. 2001: Community-oriented primary care: a model for public health nursing. Journal of Health Politics, Policy \& Law 26, 617-34.

Evans, R.G., Barer, M.L. and Marmor, T.R., editors. 1994: Why are some people healthy and others not? The determinants of health of populations. New York: Aldine de Gruyter.

Gillam, S. and Schamroth, A. 2002: The community-oriented primary care experience in the United Kingdom. American Journal of Public Health 92, 1721-25.

Keleher, H. 2001: Why primary health care offers a more comprehensive approach for tackling health inequities than primary care. Australian Journal of Primary Health 7, 57-61.

King, A. and Ministry of Health 2001: The primary health care strategy. Wellington, New Zealand: Ministry of Health.

Lopez-Acuña, D., Pittman, P., Gomez, P., Machado de Souza, H. and López Fernández, L.A. 2000: Reorienting health systems and services with health promotion criteria: a critical component of health sector reforms. Washington, DC: Pan American Health Organization.

Marriott, J. and Mable, A.L. 2000: Opportunities and potential: a review of international literature on primary health care reform and models. Ottawa: Health Canada.

Marshall, J. and Craft, K. 2000: New vision for community health services for the future. Perth: Health Department of Western Australia.

McElmurry, B.J. and Keeney, G.B. 1999: Primary health care. Annual Review of Nursing Research 17,241-68.

McElmurry, B.J., Marks, B.A., Cianelli, R. and Mamede, M. 2002: Primary health care in the Americas: conceptual framework, experiences, challenges and perspectives. Pan American Health Organization \& the World Health Organization.

McMillan, J.H. and Schumacher, S. 1997: Research in education: a conceptual introduction, fourth edition. New York: Longman.

Ministry of Health, New Zealand. 2003: Public health in a primary health care setting. Wellington, New Zealand: Public Health Directorate, Ministry of Health.

Moulton, G., Frankish, J., Rootman, I., Cole, C. and Gray, D. 2006: Building on a foundation: strategies, processes and outcomes of health promotion in primary health care settings. Primary Helath Care Research and Development 7 (in press).

National Forum of Health. 1998: Canada health action: building on the legacy papers commissioned by the National Forum of Health. Striking a balance: health care systems in Canada and elsewhere, Volume 4. Sainte-Foy, QC: National Forum of Health.

Nova Scotia Department of Health. 2001: Strengthening primary care in Nova Scotia communities: Progress report. Halifax: Nova Scotia Department of Health.

Nutbeam, D. 1998: Health promotion glossary. Health Promotion International 13, 349-64. 


\section{C James Frankish et al.}

Pearson, P. and Jones, K. 1994: The primary health care nonteam. British Medical Journal 309, 1387-88.

Swaby, M. and Biesot, A. 2001: A framework for best practice health promotion in primary health care. Australian Journal of Primary Health 7, 101-105.

Victorian Department of Human Services. 2000: Primary care partnerships: draft health promotion guidelines. Melbourne: Public Health Division and the Aged, Community and Mental Health Division.
World Health Organisation. 1978: Declaration of Alma-Ata International Conference on Primary Health Care, AlmaAta, USSR, 6-12 September 1978. Retrieved 31 March 2006 from, www.who.int/hpr/NPH/clocs/declaration_almaata.pdf

World Health Organization. 1986: Ottawa Charter for Health Promotion. Geneva: World Health Organization.

Ziglio, E., Hagard, S. and Griffiths, J. 2000: Health promotion development in Europe: achievements and challenges. Health Promotion International 15, 143-54. 